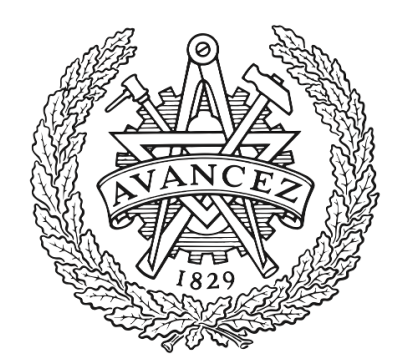

\title{
CHALMERS
}

UNIVERSITY OF TECHNOLOGY

\section{Ka-Band Gap Waveguide Coupled-Resonator Filter for Radio Link Diplexer Application}

Downloaded from: https://research.chalmers.se, 2023-04-26 08:08 UTC

Citation for the original published paper (version of record):

Alfonso Alos, E., Uz Zaman, A., Kildal, P. (2013). Ka-Band Gap Waveguide Coupled-Resonator Filter for Radio Link Diplexer Application. IEEE Transactions on Components, Packaging and Manufacturing Technology, 3(5): 870-879. http://dx.doi.org/10.1109/tcpmt.2012.2231140

N.B. When citing this work, cite the original published paper.

C2013 IEEE. Personal use of this material is permitted.

However, permission to reprint/republish this material for advertising or promotional purposes 


\title{
Ka-band Gap Waveguide Coupled-Resonator Filter for Radio Link Diplexer Application
}

\author{
Esperanza Alfonso, Member, IEEE, Ashraf Uz Zaman, and Per-Simon Kildal, Fellow, IEEE
}

\begin{abstract}
Gap waveguide technology represents an interesting alternative as low-loss, cost-effective and high-performance transmission line and package of microwave and millimeter-wave systems. A Ka-band coupled-resonator filter for a radio link diplexer, which requires high selectivity to isolate transmit and receiving channels, is proposed and realized using gap waveguide technology. The band-pass filter, which has a central frequency of $37.37 \mathrm{GHz}$ and a pass bandwidth of $560 \mathrm{MHz}$, is fabricated between two parallel metal plates leaving an air gap between them. After milling one of the plates, silver-plating is applied on them. Measurements show a minimum in-band insertion loss of 1 dB and agree quite well with simulations.
\end{abstract}

Index Terms-Band pass filters, gap waveguides, millimeterwave circuits, microwave filters, resonator filters.

\section{INTRODUCTION}

$\mathrm{G}$ AP waveguides were first introduced in [1] as a novel transmission line technology. This guiding technology is especially interesting to realize circuits and components at frequencies over $30 \mathrm{GHz}$ up to $\mathrm{THz}$. At those frequencies, the current technologies show some deficiencies regarding to the performance or to the manufacturing complexity. Hollow waveguides are resorted for low-loss applications, but the main handicaps lay in the integration with active components and in manufacturing difficulty that result in a high-cost product. Planar technologies, such as microstrip and coplanar, are more suitable for integration and easier to fabricate, but they suffer from higher losses with increasing frequency as well as from the presence of cavity resonances when encapsulated, what entails disruption from the expected performance. Therefore, there is evidently a need for new transmission line technologies at the $\mathrm{mm}$ - and sub mm-wave bands and beyond. An example is the Substrate Integrated

Manuscript received July 4, 2012. This work was supported in part by The Swedish Governmental Agency for Innovation Systems (VINNOVA) within the VINN Excellence Center Chase and in part by the Swedish Research Council VR.

E. Alfonso is with the Antenna Group, Department of Signals and Systems, Chalmers University of Technology, Gothenburg, Sweden (e-mail: esperanza.alfonso@chalmers.se).

A. U. Zaman is with the Antenna Group, Department of Signals and Systems, Chalmers University of Technology, Gothenburg, Sweden (e-mail: zaman@chalmers.se).

P.-S. Kildal is with the Antenna Group, Department of Signals and Systems, Chalmers University of Technology, Gothenburg, Sweden (e-mail: per-simon.kildal@chalmers.se).
Waveguide (SIW) technology that has been widely investigated the last years for high-frequency applications [2][4], but it exhibits significant losses at increasing frequencies due to wave propagation in substrate. On the other hand, gap waveguides can be made of only metal with waves propagating in the air gap between two metal plates. Such waveguides have been presented as interesting candidates to become alternative guiding technology for these frequency bands [1].

Gap waveguides are made between two parallel metal plates. One of the plates is provided with a texture, in the form of a bed of nails [5], but other periodic elements can also be used [6]. This texture creates a high impedance condition at the surface (ideally, a Perfect Magnetic Conducting (PMC) surface is desired), which in turn forces a cut-off for the parallel-plate modes, i.e., wave propagation is forbidden between these two plates as far as the distance between them is smaller than $\lambda / 4$. For the purpose of providing a propagation path for the waves, metal ridges are present in between the nails, in such a way that the waves follow the ridges confined to the air gap between the ridges and the metal plate on top (see Fig. 1). This is the so-called ridge gap waveguide [1], [7]. In addition, this propagation path can also be provided by a microstrip line lying on the bed of nails, or by a groove in between the nails, giving rise to the microstrip gap waveguide, and the groove gap waveguide, respectively [8]. There exist also independent realizations of related metamaterial-based guiding structures in [9].

Waves propagate in the form of a TEM-mode following ridges/strips within the ridge and microstrip gap waveguides. For the groove gap waveguide, however, propagation is of TE-type, like in rectangular waveguides. In [10], it was shown that among these three configurations, microstrip, ridge and groove gap waveguides, groove gap waveguide resonators provide the highest quality factor $(Q)$. Therefore, resonators in groove gap waveguide have been used in the present paper to realize a coupled-resonator filter for a $38 \mathrm{GHz}$ radio link diplexer where the insertion loss requirement is a critical factor.

Microwave and RF filters are essential components for most communication systems [11]. Particularly, filters are used in the microwave links transferring signals between base stations in cellular communication systems. Fig. 2 shows a block diagram of the RF front end of a radio link. The base station is transmitting and receiving simultaneously. 


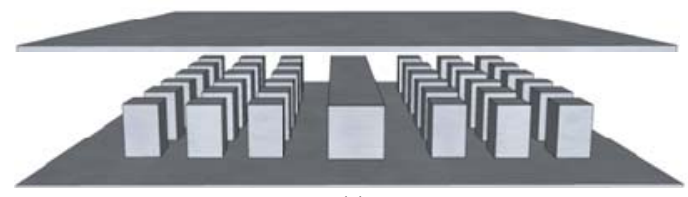

(a)

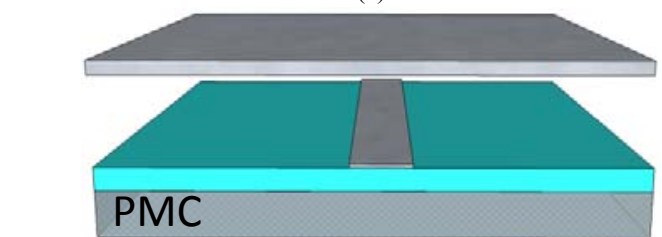

(b)

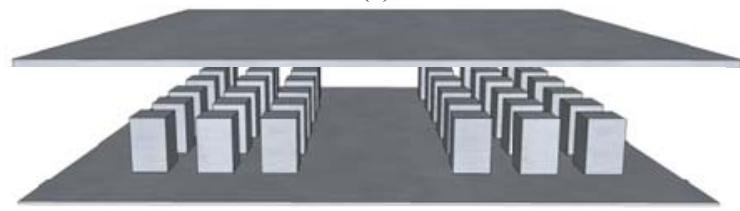

(c)

Fig. 1. Sketch of the three configurations of gap waveguides: (a) ridge, (b) microstrip, and (3) groove gap waveguides.

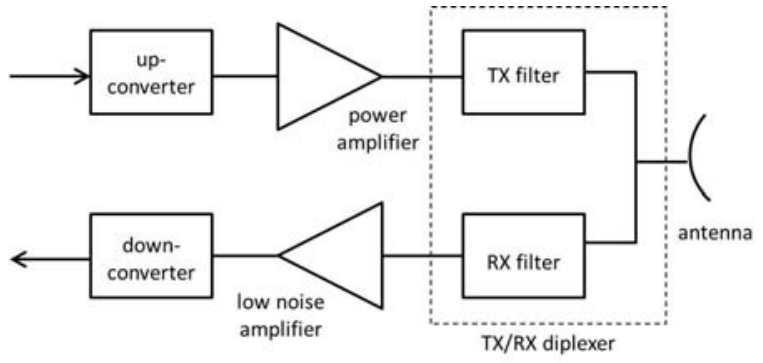

Fig. 2. RF front end of a radio link.

The diplexer is a critical component in this system, since it separates the TX and RX signals and at the same time connects them to a common antenna port. These diplex filters require very low insertion loss in the passband, and high selectivity to reject signal frequencies close to the passband. In order to fulfill such stringent requirements, these high frequency filters are normally constructed using waveguides. Traditionally, they have been designed with iris filters and Eplane metal insert filters [12] in rectangular waveguide and they represent a significant product cost. Moreover, these filters contribute to increase the size and complexity of the system, as these modules in rectangular waveguide must be connected to the electronic modules, which contain active components and MMICs, mounted on a PCB and interconnected with microstrip or coplanar lines. The RF front ends for point-to-point microwave links could benefit from the gap waveguide technology to integrate active and passive parts in the same module. On one hand, gap waveguides can be used to package microstrip circuits without creating cavity resonances, as shown in [13], [14]. On the other hand, they can be used as transmission lines to realize passive components like filters and couplers [15]-[17]. In this way, by making use of these two functionalities, gap waveguides can provide complete integration of all parts of the system. Passive and active circuits can be integrated in the same module; even the antenna can be included. Therefore, one of the main advantages of gap waveguide technology is to provide system integration between two parallel-metal plates, which do not require any conducting contact between them. As a result, the system becomes more compact and the manufacturing difficulty and cost are reduced notably.

It is worth to mention that for radio links at higher frequencies, like E-band, new technologies are needed. It is especially here, for millimeter- and sub-millimeter-wave applications, where gap waveguides represent a promising solution. The demonstration of a complete RF front end for microwave links made in gap waveguide technology is beyond the scope of this paper. Still, it is worth to note that the potential and advantages of gap waveguide technology are more outstanding when applied to a complete system than when used for a single component.

In the millimeter-wave band, substrate losses are a limiting factor for filter performance. Waveguide filters using bulk micromachining of silicon provide good performance due to high fabrication accuracy [18]-[20]. However, most of these filters use standard waveguide input/output interfaces that can limit their applications. A recent approach has been proposed to integrate filters on top of wafers [21]. This approach eliminates the dielectric loss by elevating cavity-based filters into the air with the aid of the polymer-core conductor surface micromachining technology. Maximum unloaded $Q$ of 645 for the resonators and insertion loss of $2.45 \mathrm{~dB}$ for a four-pole transmission-zero filter were observed at $60 \mathrm{GHz}$. However, the use of a thick polymer SU-8 makes this approach very sensitive to high temperature and pressure. Another integration method is found in [22]-[23]. The method presented in [22] is based on etching the backside of lossy silicon substrate underneath the short-circuited coplanar waveguide (CPW) coupling area. However, substrate losses and transition losses between a thin-film microstrip (TFMS) line and a CPW line are not completely suppressed. This problem was improved in [23], where a W-band four-pole band-pass filter was presented. A minimum insertion loss of $1.3 \mathrm{~dB}$ was obtained for a $4.9 \% 3-\mathrm{dB}$ fractional bandwidth.

The purpose of this paper is to demonstrate whether gap waveguide technology can be used for the narrow-band bandpass diplex filters used for radio links at $38 \mathrm{GHz}$. The stringent specifications of these filters regarding to the low loss and high roll-off require around eight resonators with unloaded $Q$ of the order of $10^{3}$ [11]. So far, to the author's knowledge, only filters made in waveguide can provide such high values of $Q$. Since groove gap waveguide resonators have been shown to provide values of $Q$ comparable to those provided by rectangular waveguides [10], it should be possible to design a $38 \mathrm{GHz}$ radio link filter in groove gap waveguide technology. Section II shows the filter specifications. Section III describes the design process of coupled-resonator filters and how to calculate the filter parameters required for this particular design. The relationship 
between filter parameters and filter geometry is established in Section IV, through design curves. Couplings between resonators as well as tuning are done in different ways from those used in [17]. Finally, simulated and measured results are shown in Section V.

\section{Filter SPECIFICATIONS}

The specifications for the $38 \mathrm{GHz}$ radio link diplex filter are shown in Table I.

TABLE I

\begin{tabular}{ll}
\multicolumn{2}{c}{ SPECIFICATIONS FOR THE $38 \mathrm{GHz}$ DiPLEX FILTER } \\
\hline \hline Passband & $37.058-37.618 \mathrm{GHz}$ \\
Stopband & $38.318-38.878 \mathrm{GHz}$ \\
Insertion loss & $1.5 \mathrm{~dB}$ \\
Attenuation & $70 \mathrm{~dB}$ \\
Return loss & $-17 \mathrm{~dB}$ \\
\hline \hline
\end{tabular}

The above specifications correspond to a fractional bandwidth (BW) of 0.015 , and involve a steep roll-off to reject signal frequencies within the stopband. The selectivity of a filter increases with the number of resonant sections. Furthermore, the insertion loss in the passband is inversely proportional to the filter bandwidth and the resonator $Q$, and is proportional to the number of resonators used.

The design of this filter is described in the following section. A general technique for designing coupled-resonator filters has been used. It can be applied to any kind of resonator despite its physical structure. This technique is based on coupling coefficient of intercoupled resonators and the external quality factors of the input and output resonators. These parameters can be obtained numerically from the frequency response of resonators using an electromagnetic solver [24].

\section{FILTER DESIGN}

Any narrow-band, lumped-element, or distributed band-pass filter can be described by three fundamental variables: the synchronous tuning frequency of each resonator, $f_{0}$; the coupling between adjacent resonators, $K$; and the singly loaded or external $Q$ of the first and last resonators, $Q_{e x}[25]$. The general band-pass filter design procedure utilizes the normalized, low-pass elements of the prototype filter to determine the required coupling coefficient between resonators and the coupling to the external circuit; that is, the source and load. The external source and load are assumed to be $50 \Omega$, although the filter could be matched to other real impedances. The low-pass prototype filter is a lumped element network that is synthesized to provide a desired filter transfer function. In this case, a Chebyshev response with the parameters shown in Table II was chosen. As usual practice, a bandwidth $20 \%$ wider than the required $1.5 \%$ has been considered for the design.
TABLE II

FILTER PARAMETERs FOR CHEBYSHEV RESPONSE

\begin{tabular}{ll}
\hline \hline Fractional bandwidth & $1.8 \%$ \\
Attenuation in the stopband & $70 \mathrm{~dB}$ \\
Maximum ripple in the passband & $0.01 \mathrm{~dB}$ \\
\hline \hline
\end{tabular}

From the filter parameters for Chebyshev response of Table II, the graphs and equations in [26] were used to obtain an estimation of the filter order $\mathrm{N}$, and the corresponding normalized low-pass prototype element values for an $\mathrm{N}^{\text {th }}$ order filter $(\mathrm{N}=7$ for this case):

$$
\begin{array}{ll}
g_{0}=g_{8}=1 & g_{3}=g_{5}=1.7481 \\
g_{1}=g_{7}=0.7969 & g_{4}=1.6331 \\
g_{2}=g_{6}=1.3924 &
\end{array}
$$

From the low-pass parameters, the required coupling coefficients $(K)$ and external quality factors $\left(Q_{e x}\right)$ are calculated as [25]:

$$
\begin{gathered}
K_{i, i+1}=\frac{\mathrm{BW}}{\sqrt{g_{i} g_{i+1}}}, \quad i=1, \ldots, N-1 \\
Q_{e x_{i}}=\frac{g_{i} g_{i+1}}{\mathrm{BW}}, \quad i=0, N
\end{gathered}
$$

Thus, the values obtained for the coupling coefficients are:

$$
\begin{array}{ll}
K_{01}=K_{78}=0.0202 & K_{23}=K_{56}=0.0115 \\
K_{12}=K_{67}=0.0171 & K_{34}=K_{45}=0.0107
\end{array}
$$

And the value for the external quality factor of the input and output resonators is $Q_{e x}=44.27$.

At this point, we can estimate the minimum average unloaded $Q$ for the resonators using the insertion loss specification in table I from the formula in [25]:

$$
\operatorname{Loss}\left(f_{0}\right)=\frac{4.343 f_{0}}{\mathrm{Q}_{u} \mathrm{BW}} \sum_{i=1}^{N} g_{i}(d B)
$$

where $\operatorname{Loss}\left(f_{0}\right)$ is the insertion loss at the center frequency, and $Q_{u}$ is the unloaded $Q$. This gives a minimum $Q_{u}=1837$ for the resonators to fulfill filter specifications.

Once the band-pass filter parameters are known, the next step of the design process is to establish the relationship between these parameters and the filter geometry. For this purpose, design curves relating the filter parameters $\left(K\right.$ and $\left.Q_{e x}\right)$ to physical parameters of the resonators were calculated, as described in the next section.

\section{Design CuRves}

In order to determine the filter geometry, design curves have to be previously calculated. Such curves describe the 
relationship between the filter parameters, i.e., coupling coefficient between resonators $(K)$ and coupling to the external network or external quality factor $\left(Q_{e x}\right)$, and some parameters describing the filter geometry.

Two resonant peaks are observable from the frequency response of a pair of coupled resonators (see Fig. 3). Once the natural resonant frequencies $\left(f_{\text {high }}, f_{\text {low }}\right)$ of these two peaks are found, the coupling coefficient of two synchronously coupled resonators is calculated as

$$
K=\frac{f_{\text {high }}^{2}-f_{\text {low }}^{2}}{f_{\text {high }}^{2}+f_{\text {low }}^{2}}
$$

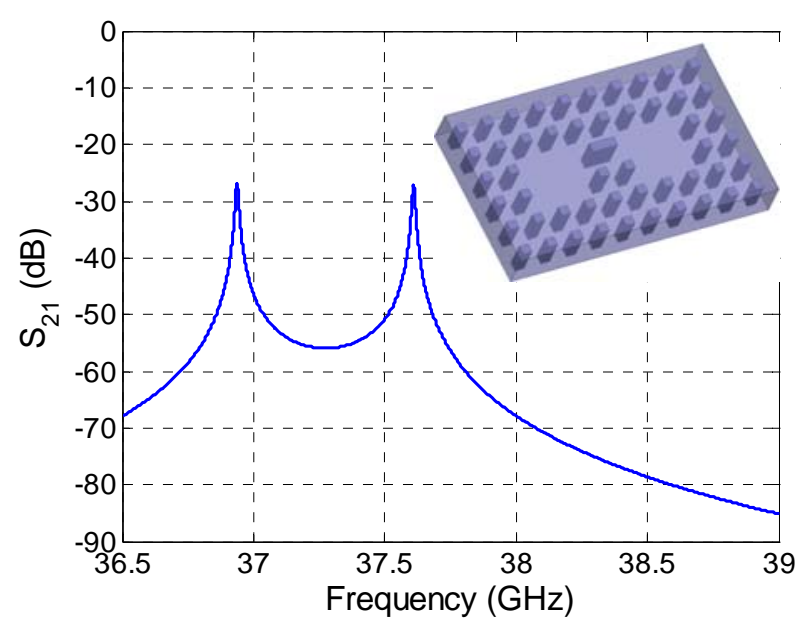

Fig. 3. Frequency response of a pair of coupled resonators.

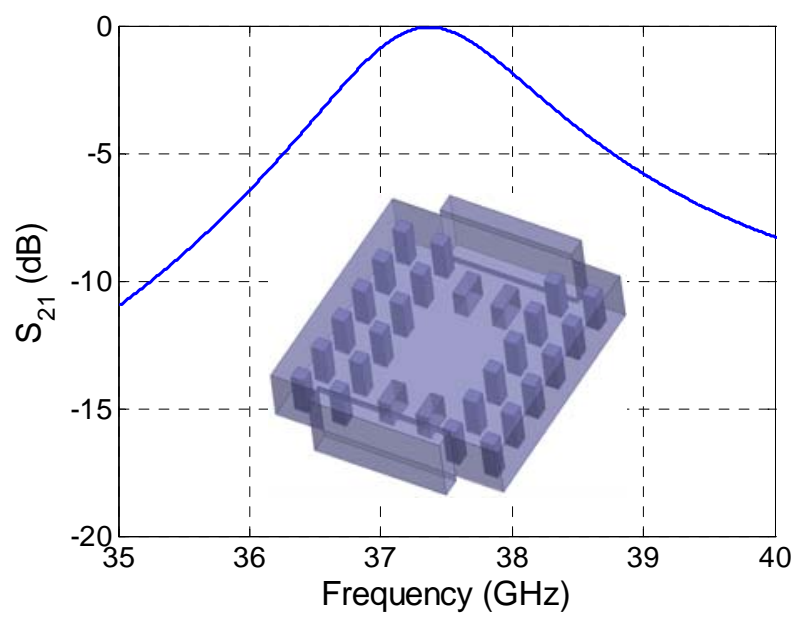

Fig. 4. Frequency response of a doubly-loaded resonator.

For measurement purposes, the input and output resonators are connected to standard Ka-band WR-28 rectangular waveguide flanges. The external quality factor can be obtained from the frequency response of a doubly-loaded resonator (see Fig. 4) as

$$
Q_{e x}=\frac{2 \cdot f_{0}}{B W_{-3 d B}}
$$

where $B W_{-3 d B}$ is the bandwidth at $-3 \mathrm{~dB}$.

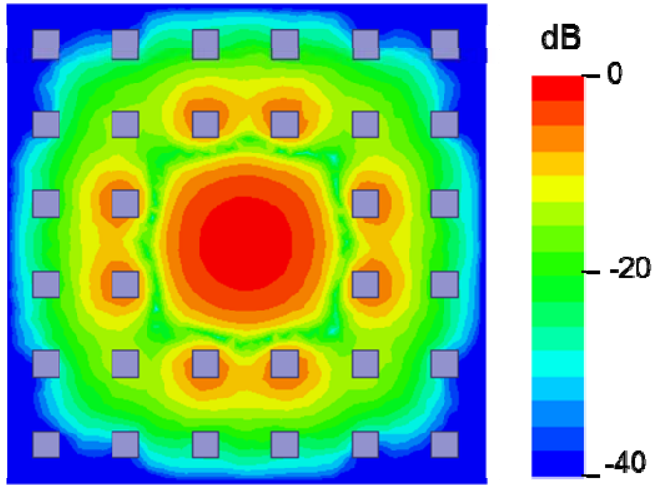

Fig. 5. Electric field pattern of one resonator in groove gap waveguide.

The design procedure is as follows. Square cavities in groove gap waveguide were chosen. These cavities are not closed structures; however they work in the same way as completely closed resonators, i.e., they show a similar electromagnetic field pattern, as depicted in Fig. 5. The cavities are created in the region formed by the groove in between the nails on one of the plates and the flat metal plate on top. Fields are confined to the groove region without the need of any metal contact between the two metal plates. The bed of nails imposes a cut-off for the parallel-plate modes in the air gap between the two plates, so that fields cannot expand through this gap, and therefore they remain confined to the cavity or groove region. It has been shown that attenuation is around 20 $\mathrm{dB}$ per row of nails [7], thus, waves are completely attenuated after two rows of nails, more than $40 \mathrm{~dB}$.

First, only one single isolated resonator is considered. Its size is tuned to show one mode resonating at a frequency equal to the central frequency of the filter pass-band. Resonators of 5.6 $\times 5.6 \mathrm{~mm}^{2}$ show a resonant frequency at $37.37 \mathrm{GHz}$. Dimensions of the bed of nails and air gap height have been chosen to show a stopband for the parallel-plate modes between 25 and $50 \mathrm{GHz}$, following the design rules in [1]. Length of the nails must be around $\lambda / 4$, and air gap height below $\lambda / 4$. The smaller the air gap height is, the larger the bandwidth is. Thickness of the nails and grid period are not decisive for the bandwidth, as far as the period is smaller than $\lambda / 2$. Nails have square shape and they are $0.7 \mathrm{~mm}$ thick and $2.3 \mathrm{~mm}$ long. Period of nails is $2.1 \mathrm{~mm}$, and air gap height is $0.5 \mathrm{~mm}$.

The unloaded $Q$ of these resonators is 3365 considering silver as metal and air gap height $0.5 \mathrm{~mm}$. This value is over the minimum required unloaded $Q$ for the resonators, which was estimated from (5). Higher values of the unloaded $Q$ can be obtained for larger air gap heights, as can be seen in Table III, 
but at the expense of a reduction of the parallel-plate mode stopband.

TABLE III

UNLOADED Q OF A RESONATOR

\begin{tabular}{cc}
$\begin{array}{c}\text { Air gap } \\
\text { height }(\mathrm{mm})\end{array}$ & $\begin{array}{c}\text { Unloaded } \\
\mathrm{Q}\end{array}$ \\
\hline \hline 0.25 & 3248 \\
0.5 & 3365 \\
0.75 & 3429 \\
1 & 3492 \\
1.25 & 3632 \\
1.5 & 3771 \\
\hline \hline
\end{tabular}

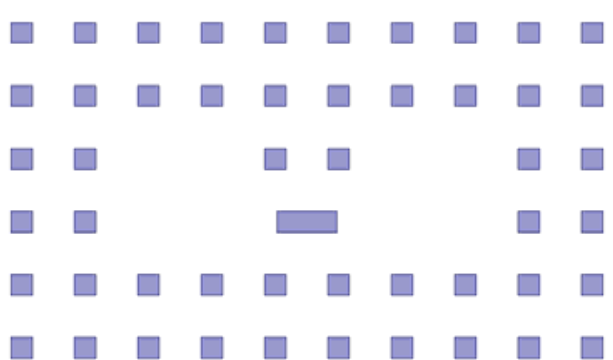

Fig. 6. Nails pattern of two coupled resonators in groove gap waveguide. The ridge increases the coupling level between them.

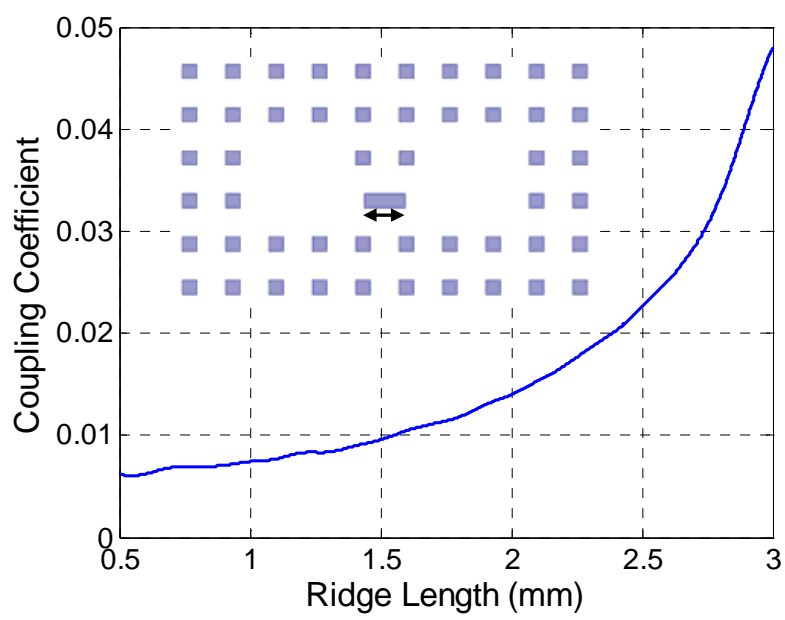

Fig. 7. Coupling coefficient between two coupled resonators as a function of the ridge length. Ridge height is $2.3 \mathrm{~mm}$, the same as nails' length.

Next, for the study of the coupling coefficient $(K)$ between adjacent resonators, two resonators are placed side by side in such a way that adjacent cavities are separated by two rows of nails [17]. The cavities are loosely excited. The coupling coefficient is calculated from the frequency response using (6). A low value of the coupling coefficient compared to the ones required for the design and given by (4) is obtained in this way. In order to increase the coupling between resonators, two nails are replaced by a ridge of the same width as the nails' thickness, as shown in Fig. 6. This coupling has been varied by changing ridge's size, as can be seen in Fig. 7 and Fig. 8, where the coupling coefficient has been plotted as a function of the ridge length, and ridge height, respectively. Those plots are the design curves for the coupling coefficient $(K)$ between resonators that will be used to determine the sizes of the ridges connecting such resonators.

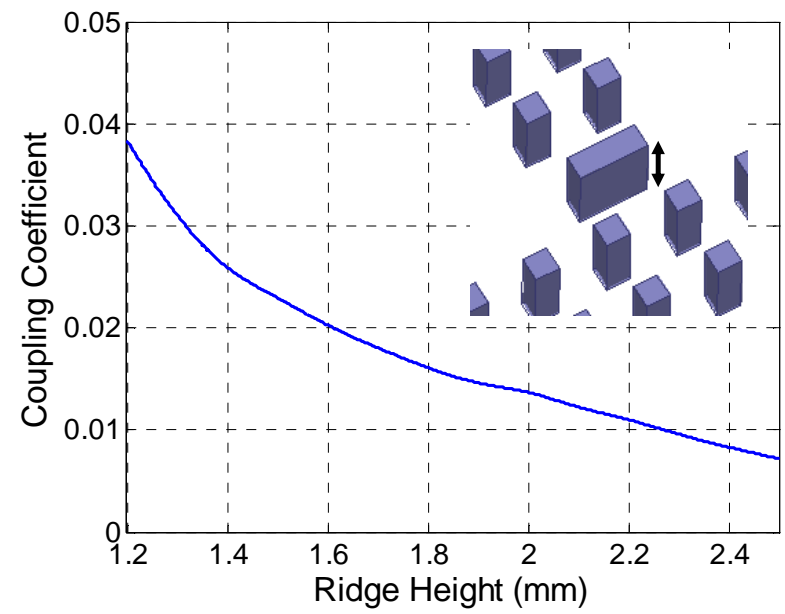

Fig. 8. Coupling coefficient between two coupled resonators as a function of the ridge height. Ridge length is $1.5 \mathrm{~mm}$.

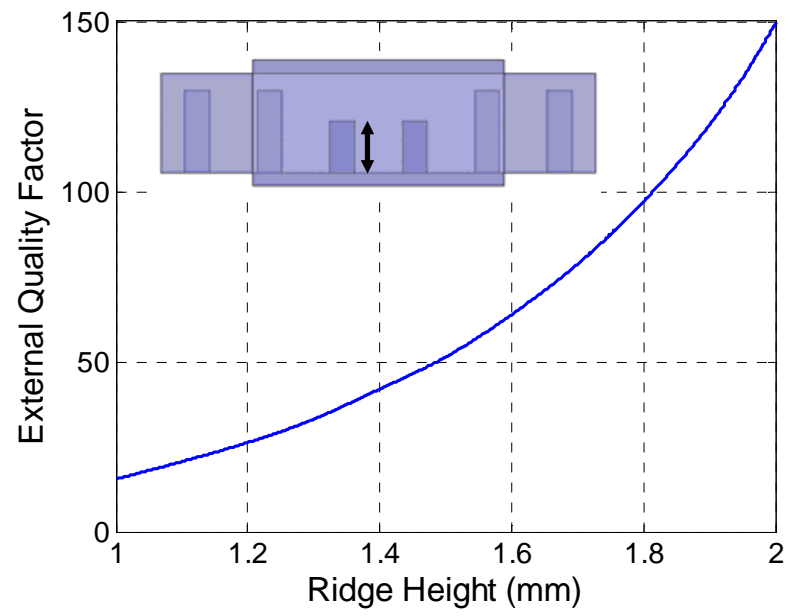

Fig. 9. External quality factor of one resonator doubly-loaded by standard Kaband WR-28 rectangular waveguide as a function of ridges' height. Two ridges as coupling elements between the resonator and the external circuit or load. Ridges' position is $-0.3 \mathrm{~mm}$ and ridges' length is $1.5 \mathrm{~mm}$.

Then, the input and output resonators must be loaded, i.e., coupled to the external network, in a proper way, so that values of $Q_{e x}$ around 44.27 are provided. For measurement purposes, a WR-28 rectangular waveguide is connected to the input and output resonators. Two ridges are now used as coupling elements from input/output resonators to the external network. It was assumed that the two ridges are identical, and their width is equal to the nails' thickness. By varying the ridges' height it is possible to adjust the external quality factor to the design value, but the resonant frequency is slightly affected as well. With the aim of tuning the frequency, two physical parameters have been found to be appropriate for tuning, since those parameters hardly has an effect on $Q_{e x}$. 
One of them is the ridges' position in the direction parallel to the ridges, and the other one is the ridges' length. The variation of the resonant frequency as a function of ridges' position and ridges' length is shown in Fig. 10 and Fig. 11 respectively. For the range of values represented in Fig. 11 for the ridges' length, the $Q_{e x}$ is kept almost constant. From Figs. 9-11 the physical parameters of these two ridges (height, length, and position in the direction parallel to the ridge) can be determined to get a $Q_{e x}=44.27$ at a central frequency $f_{0}=37.37 \mathrm{GHz}$.

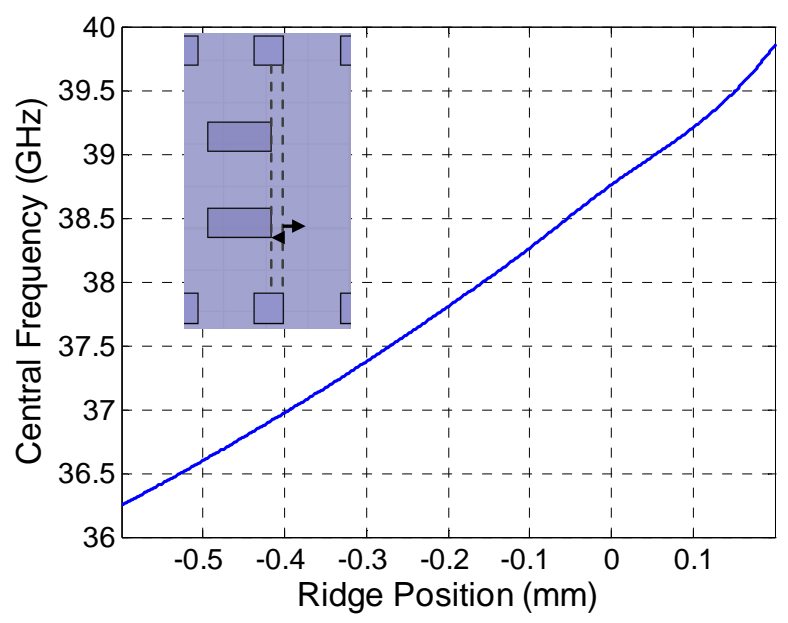

Fig. 10. Central frequency of a doubly-loaded resonator as a function of ridges' position. Ridges' height is $1.43 \mathrm{~mm}$ and ridges' length is $1.5 \mathrm{~mm}$.

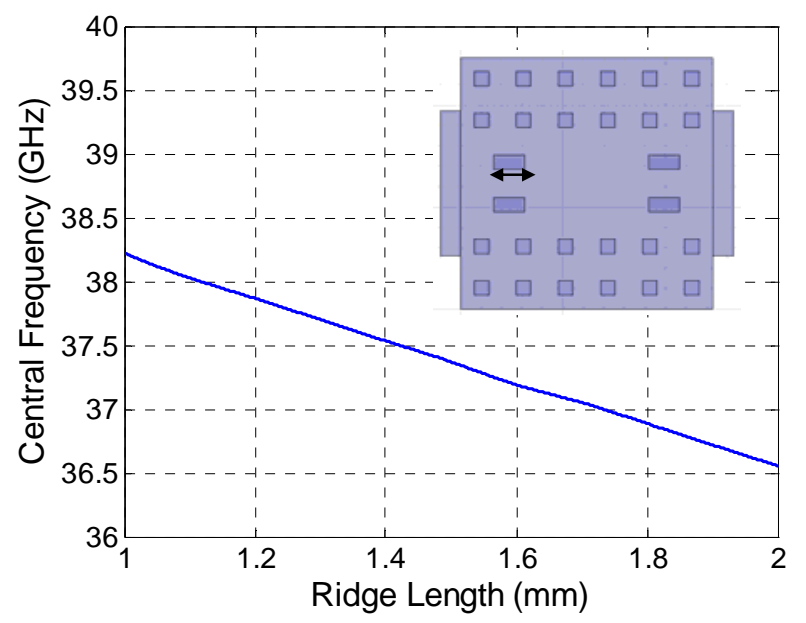

Fig. 11. Central frequency of a doubly-loaded resonator as a function of ridges' length. Ridges' position is $-0.3 \mathrm{~mm}$ and ridges' height is $1.43 \mathrm{~mm}$.

\section{RESUlts}

Once the values of filter design parameters $\left(K\right.$ and $\left.Q_{e x}\right)$ have been obtained from the filter specifications and the design curves relating those parameters with the filter geometry have been calculated, the initial filter design can be obtained.

As it is usual when several resonators are placed side by side in cascade, couplings between resonators as well as resonant frequencies of each resonator are changed from the ones obtained when only two resonators are considered. Therefore, it is always needed an optimization stage to get satisfactory results. This is the case here, where the filter pass-band appears at lower frequencies and the return loss requirement is not satisfied. First, the initial design is scaled down to move the pass-band to higher frequencies. But still, resonant frequencies and couplings have to be tuned to fulfill the specified return loss within the pass-band. Couplings between resonators can be controlled by varying ridges' lengths. Resonant frequencies can be adjusted by means of cavity sizes. Cavity width (size of the cavities in longitudinal direction) is considered fixed, and cavity lengths are varied. From the manufacturing point of view, it is better to keep uniform the grid of nails, thus, in order to increase cavity lengths the pins located in longitudinal direction at the border of the cavities have been slightly sawed so that the groove size in transverse direction becomes larger. Only a few microns were enough, so in practice pin size is hardly modified. Since a symmetric design was supposed, a total of seven parameters were taken into account for the optimization, three parameters corresponding to ridge lengths, and four parameters corresponding to cavity lengths.

Fig. 12 shows a sketch of the filter obtained after optimization; it can be appreciated the uniform distribution of squared nails and the ridges interconnecting adjacent resonators and end resonators to the external network (WR28). Nails and ridges are located on the bottom metal plate. Note that the reduced size of the pins at the border of cavities of only few microns is barely perceptible.

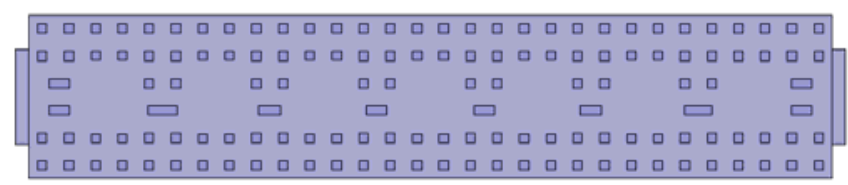

Fig. 12. Sketch of the optimized coupled-resonator filter in groove gap waveguide technology.

\section{A. Simulated results}

Fig. 13 shows the frequency response of the optimized filter. HFSS was used for the calculations, and silver as conducting metal was considered in simulations. As can be seen, specifications are satisfied. Return loss better than $-17 \mathrm{~dB}$ and insertion loss less than $1.5 \mathrm{~dB}$ within the pass-band were obtained. Isolation higher than $70 \mathrm{~dB}$ within the stop-band is also satisfied. Insertion loss at the central frequency is below 1 dB.

It is worth mentioning that even though $20 \%$ wider bandwidth was considered for the design, as shown in Table II, it was not possible to meet specifications keeping such bandwidth in the optimization process, which was carried out using a general electromagnetic solver such as HFSS. Therefore, the filter design was optimized to satisfy the 
diplexer filter specifications in Table I.

The use of a more efficient and specific software for filter design is needed for the optimization of high-order filters. This will be considered in future designs.

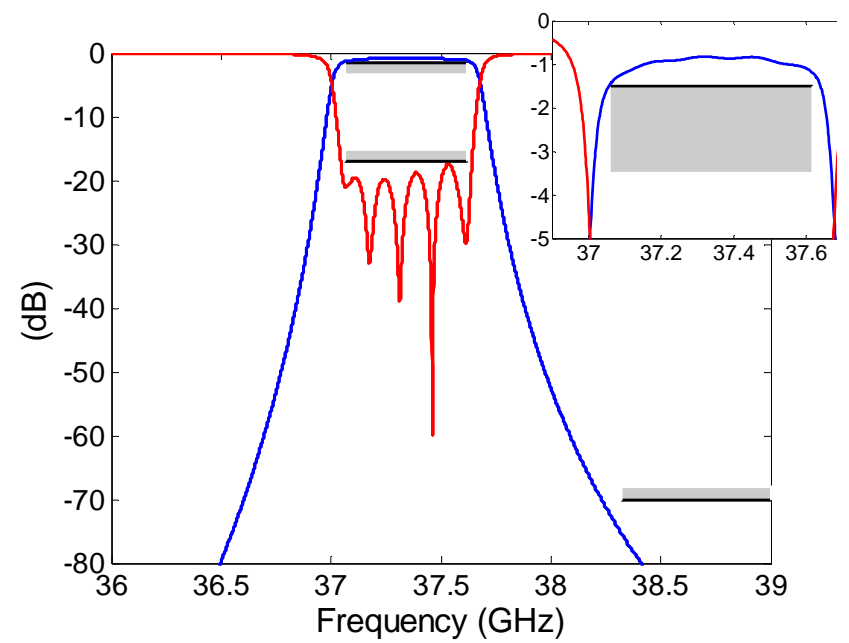

Fig. 13. Filter response of the optimized design. Specifications are also shown.

\section{B. Measured results}

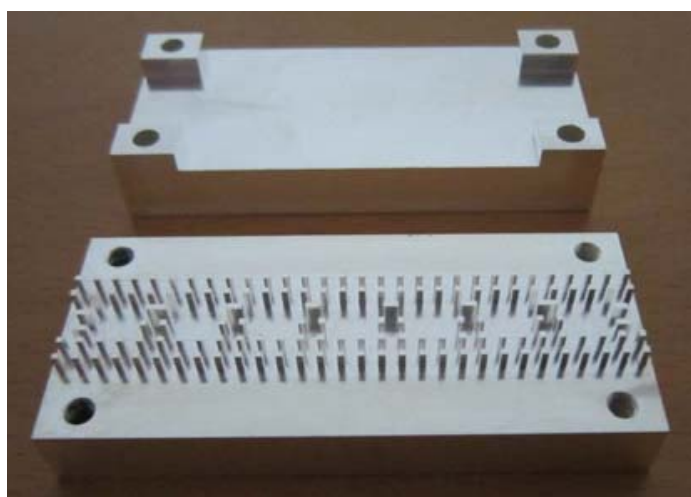

(a)

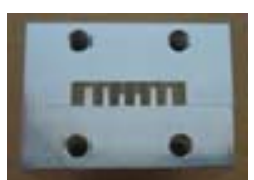

(b)

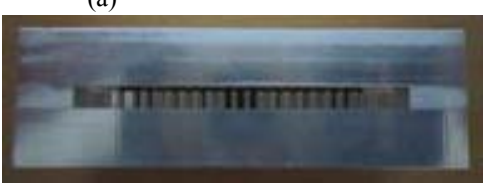

(c)
Fig. 14. Photos of the filter prototype: (a) Top and bottom plates (with holes to assemble both parts), (b) Front view (with holes to connect rectangular waveguide flanges), (c) Side view (with no sidewalls).

Two prototypes of the designed filter were manufactured; one was made of aluminum and the other one was silver-plated. The filter was made between two metal plates. One of the plates was milled to create the pins and ridges; whereas the other plate was placed above it keeping a certain distance between them, i.e., an air gap. Hence, this filter has no sidewalls (see Fig. 14). Such a property can be an advantage with respect to other cavity-based filters, as it allows airing and cooling, and in consequence it can be less sensitive to temperature drift. The fact of not requiring conducting contact between plates is also an advantage, already at this frequency, but especially at higher frequencies where the leakage through joints becomes more important.

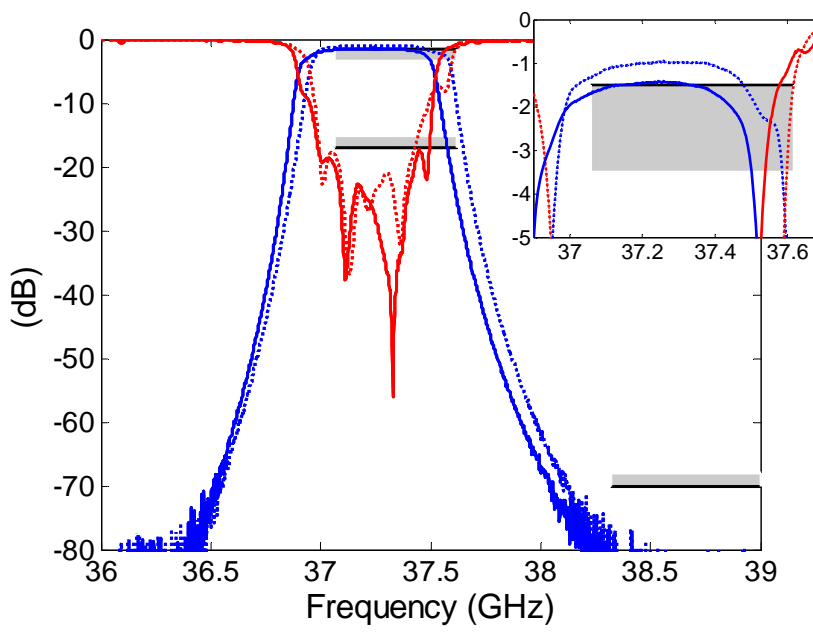

Fig. 15. Prototype responses: aluminum (solid line) and silver-plated (dotted line).

Fig. 15 shows the comparison of the filter responses given by both prototypes. It is clear from the graph that the experimentally realized pass-band shifted by about $60 \mathrm{MHz}$ towards higher frequency due to the silver-plating. This difference is caused by the silver plating of 1-2 microns thickness, which theoretically corresponds to a shift of 30 MHz. At the zoom of the pass-band shown in Fig. 15, it can be seen that there is a difference of $0.5 \mathrm{~dB}$ for the insertion loss of both responses, being higher the one given by the aluminum prototype, as expected. At the central frequency, the insertion loss is $1.5 \mathrm{~dB}$ for the aluminum prototype, and 1 $\mathrm{dB}$ for the silver-plated prototype.

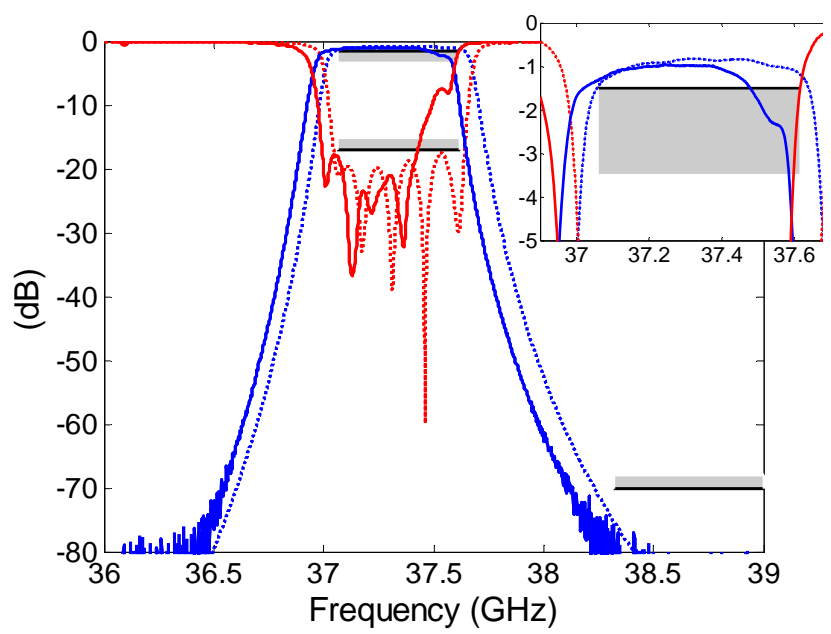

Fig. 16. Silver-plated filter responses: measured (solid line) and simulated (dotted line). 


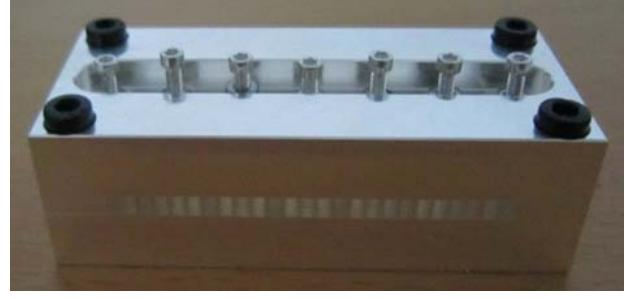

Fig. 17. Photo of the filter prototype with lid with holes in it for inserting tuning screws.

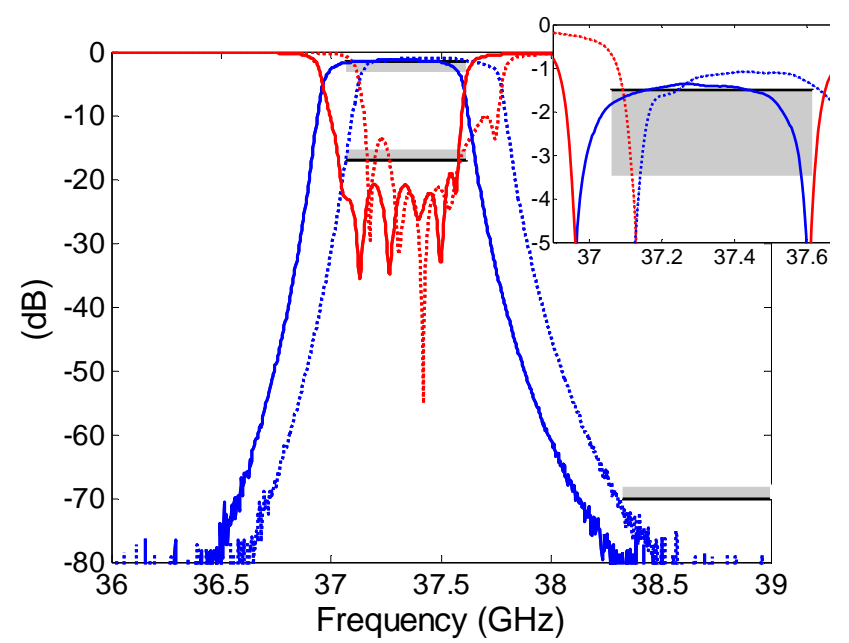

Fig. 18. Silver-plated prototype responses: using lid with holes in it before(dotted line) and after tuning (solid line).

The comparison between simulations and measurements for the silver-plated prototype is shown in Fig. 16. The measured pass-band is moved approximately $75 \mathrm{MHz}$ to lower frequencies with respect to simulated one. The difference in this case is due to manufacturing tolerances, since the milling technique used has an accuracy of $\pm 30 \mu \mathrm{m}$. Regarding the insertion loss at the central frequency simulated and measured results differ only by $0.1 \mathrm{~dB}$. The results show that the measured return loss is $-17 \mathrm{~dB}$ compared to a simulated value of $-20 \mathrm{~dB}$, due to degradation of one of the poles. Still, the results are quite satisfactory. But, it is worth to note it would have been possible to get better performance within the working pass-band if the design had been optimized for $20 \%$ wider bandwidth using efficient filter software based on mode matching or other efficient techniques.

Nevertheless, it is worth to resort to one the most common techniques for tuning narrow-band filters after manufacturing and check how it works for gap waveguide filters. It involves the use of screws, one for each cavity, to tune the resonant frequency of each cavity and in turn to move the filter passband. The screws are inserted in the cavities through some holes made on the top metal plate at the center of each resonator (see Fig. 17). The responses of the silver-plated prototype using this lid before and after tuning are shown together in Fig. 18 for comparison. It is clearly seen that the pass-band can be shifted by using tuning screws while keeping the return loss to the required level. The insertion loss is affected by the presence of screws by an increase of 0.25 $\mathrm{dB}$.

\section{Comparison with other filters}

It is important to compare the performance of the proposed gap waveguide filter with other waveguide filters used in diplexers for microwave links at $38 \mathrm{GHz}$.

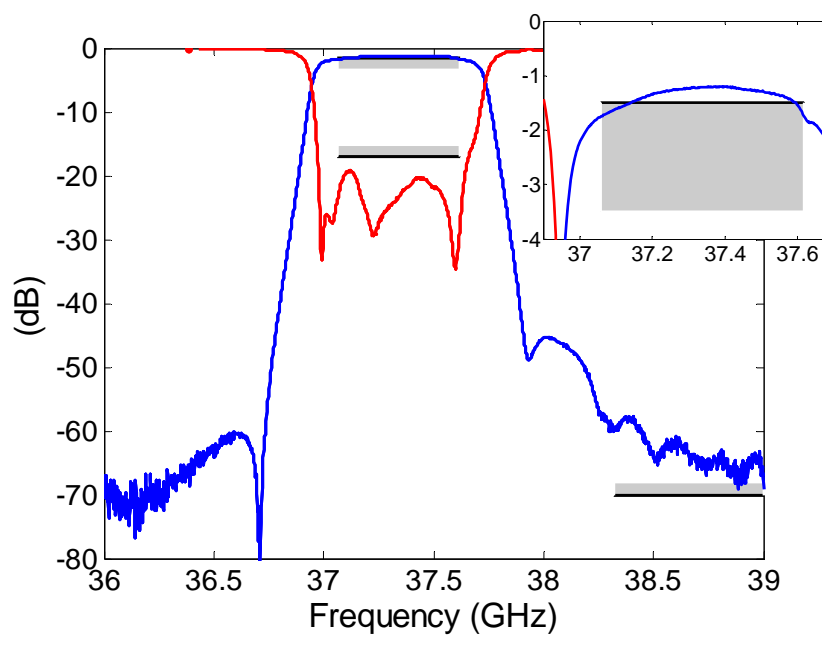

Fig. 19. Filter response of a prototype made in rectangular waveguide and used in diplexers for microwave links at $38 \mathrm{GHz}$.

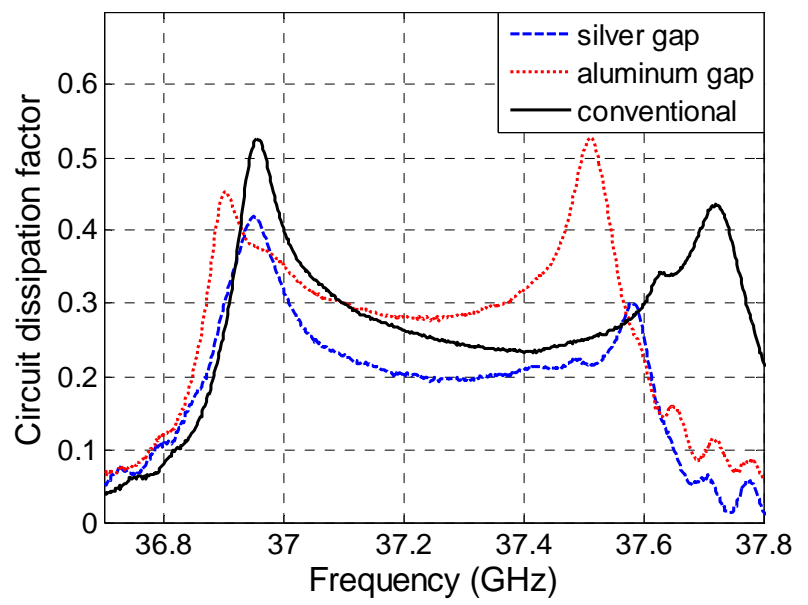

Fig. 20. Circuit dissipation factor of the silver and aluminum groove gap waveguide filters and the conventional rectangular waveguide filter.

A filter made using standard rectangular waveguide cavities and tuning screws with the same number of poles than the proposed filter was measured for comparison. As can be seen in Fig. 19, despite this filter has not so good rejection at the stopband and it has wider bandwidth than the proposed gap waveguide filter, the insertion loss is $1.3 \mathrm{~dB}$, approximately the same as the one exhibit by the gap waveguide filter when screws are used for tuning the filter after manufacturing.

Dissipative losses of the silver groove gap waveguide filter prototype are below those shown by the aluminum prototype and below the losses exhibit by the filter in conventional 
rectangular waveguide. This can be observed in Fig. 20, where the circuit dissipation factor defined as $1-\left|S_{11}\right|^{2}-\left|S_{21}\right|^{2}$ is plotted for three filters, the silver and aluminum prototypes of the groove gap waveguide filter, and the filter in conventional rectangular waveguide.

In order to study the temperature drift, both filters were measured at maximum and minimum temperatures of $+70^{\circ} \mathrm{C}$ and $-50^{\circ} \mathrm{C}$. Measurements corresponding to the gap waveguide filter are shown in Fig. 21. The frequency shift between maximum and minimum temperatures was $50 \mathrm{MHz}$, or $8.9 \%$ in terms of bandwidth. As for the prototype made in rectangular waveguide, the frequency shift was $10 \mathrm{MHz}$ higher, i.e., $60 \mathrm{MHz}$, or $10.7 \%$.

These results show that coupled-resonator filters in rectangular waveguide and groove gap waveguide provide very similar performance, and confirm that gap waveguide resonators are less sensitive to temperature drift.

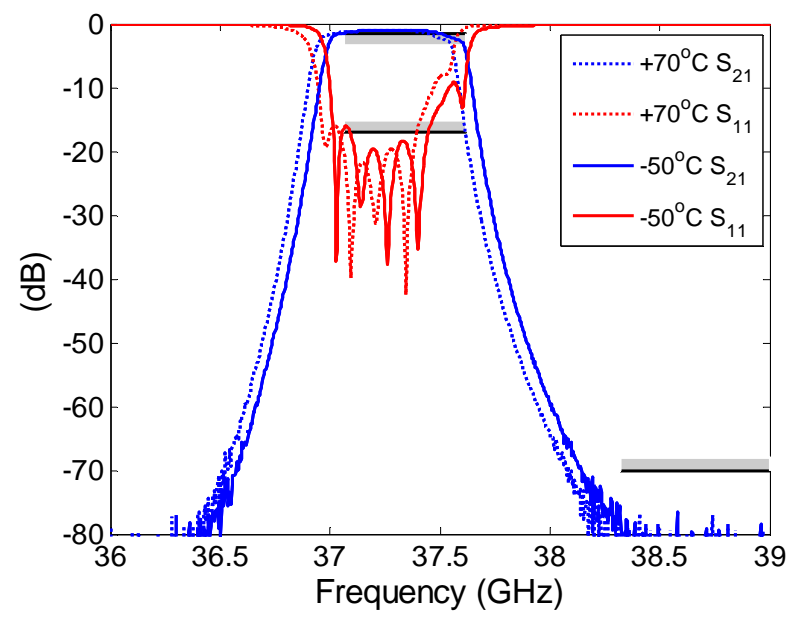

Fig. 21. Filter response of silver-plated gap waveguide filter prototype at maximum and minimum temperatures of $+70^{\circ} \mathrm{C}$ and $-50^{\circ} \mathrm{C}$.

\section{CONCLUSION}

A Ka-band coupled-resonator filter for a radio link diplexer has been proposed and demonstrated using gap waveguide technology. These narrow-band filters have very stringent requirements regarding to losses and selectivity. Groove gap waveguide resonators provide losses comparable to those shown by rectangular waveguide resonators, therefore a similar filter performance using both technologies was expected and it has been corroborated in the present paper. The prototypes shown here are only proofs of concept of realizing diplex filters in gap waveguide technology. Closer results to filter specifications for the whole pass-band could have been obtained with more accurate milling or taking into account manufacturing tolerances in the design. Filter design has been done for cost-effective manufacture, keeping a regular grid of nails. In order to tune the resonant frequency of each resonator, the size of the cavities must be varied. This has been done by simply sawing a few microns the internal face of the pins surrounding the cavities, thereby keeping the grid uniformity.

Waveguide filters are high-performance, but difficult to integrate with active components. Gap waveguide technology, however, can be used to construct passive components, such as filters, couplers, and power dividers, as well as packaging of MMICs and microstrip circuits, and thus, providing system integration between two parallel- metal plates without any conducting joint needed between them. This is the main advantage of gap waveguide technology, especially at millimeter-wave bands where mature technologies are not always appropriate.

Coupled-resonator filters in groove gap waveguide are costeffective, since they are formed in the gap between two parallel-metal plates. And moreover, they are less sensitive to temperature drift, as they are based on groove gap waveguide resonators, i.e., open cavities that allow airing and cooling.

\section{REFERENCES}

[1] P.-S. Kildal, E. Alfonso, A. Valero-Nogueira, and E. Rajo-Iglesias, "Local metamaterial-based waveguides in gaps between parallel metal plates, " IEEE Antennas and Wireless Propagation Letters, vol. 8, pp. 84-87, 2009

[2] D. Deslandes and $\mathrm{K}$. Wu, "Integrated microstrip and rectangular waveguide in planar form," IEEE Microwave and Wireless Components Letters, vol. 11, no. 2, pp. 68-70, Feb. 2001

[3] J. Hirokawa, M. Ando, and N. Goto, "Waveguide-fed parallel plate slot array antenna," IEEE Trans. Antennas Propagat., vol. 40, no. 2, pp. 218223, Feb. 1992

[4] Y. Cheng, P. Chen, W. Hong, T. Djerafi, and K. Wu, "Substrateintegrated-waveguide beamforming networks and multibeam antenna arrays for low-cost satellite and mobile systems," IEEE Antennas and Propagation Magazine, vol.53, no.6, pp.18-30, Dec. 2011

[5] M. Silveirinha, C. Fernandes, and J. Costa, "Electromagnetic characterization of textured surfaces formed by metallic pins," IEEE Trans. Antennas Propagat, vol. 56, no. 2, pp. 405-415, Feb. 2008.

[6] E. Rajo-Iglesias, P.-S. Kildal, "Numerical studies of bandwidth of parallel plate cut-off realized by bed of nails, corrugations and mushroom-type EBG for use in gap waveguides", IET Microwaves, Antennas \& Propagation, vol. 5, no. 3, pp. 282-289, March 2011.

[7] P.-S. Kildal, A. Zaman, E. Rajo-Iglesias, E. Alfonso, and A. ValeroNogueira, "Design and experimental verification of ridge gap waveguide in bed of nails for parallel-plate mode suppression," IET Microwaves, Antennas Propagat., vol. 5, no. 3, pp. 262-270, Feb. 2011.

[8] P.-S. Kildal, "Three metamaterial-based gap waveguides between parallel metal plates for $\mathrm{mm} / \mathrm{submm}$ waves," in European Conference Antennas Propagat 2009 (EuCAP), Mar. 2009, pp. 28-32.

[9] H. Kirino, K. Ogawa, "A $76 \mathrm{GHz}$ multi-layered phased array antenna using a non-metal contact metamaterial waveguide," IEEE Trans. Antennas Propagat., vol. 60, no. 2, pp. 840-853, Feb. 2012.

[10] E. Pucci, A. Uz Zaman, E. Rajo-Iglesias, P-S Kildal, and A. Kishk, "Study of Q-factors of ridge and groove gap waveguide resonators," IEEE Trans. Components, Packaging and Manufact. Tech., submitted for publication.

[11] I. C. Hunter. Theory and design of microwave filters. IET electromagnetic wave series no. 48, 2006, ch. 1.

[12] R. Vahldieck, and W.J.R Hoefer, "Finline and metal insert filters with improved passband separation and increased stopband attenuation," IEEE Trans. Microw. Theory Tech., vol.33, no.12, pp. 1333-1339, Dec. 1985.

[13] E. Rajo-Iglesias, A. Zaman, and P.-S. Kildal, "Parallel plate cavity mode suppression in microstrip circuit packages using a lid of nails," IEEE Microw. Wireless Compon. Lett., vol. 20, no. 1, pp. 31-33, Jan. 2010.

[14] A. Algaba Brazález, A. Uz Zaman, and P-S Kildal, "Improved microstrip filters using PMC packaging by lid of nails," IEEE Trans. Components, Packaging and Manufact. Tech., Jul. 2012.

[15] E. Alfonso, M. Baquero, P.-S. Kildal, A. Valero-Nogueira, E. RajoIglesias, and J.I. Herranz, "Design of microwave circuits in ridge-gap 
waveguide technology," Microwave Symposium Digest (MTT), 2010 IEEE MTT-S International, pp.1544-1547, May 2010

[16] E. Alfonso, M. Baquero, A. Valero-Nogueira, J. I. Herranz, and P.-S. Kildal, "Power divider in ridge gap waveguide technology," in European Conference Antennas Propagat 2010 (EuCAP), Apr. 2010.

[17] A. Uz Zaman, P.-S. Kildal and A. Kishk, "Narrow-Band microwave filter using high $\mathrm{Q}$ groove gap waveguide resonators with manufacturing flexibility and no sidewalls," in IEEE Trans. Components, Packaging and Manufact. Tech., vol.2, no.11, pp.1882-1889, Nov. 2012.

[18] J. Papapolymerou, J.-C. Cheng, J. East, and L. P. B. Katehi, "A micromachined high-Q X-band resonator," in IEEE Microw. Guided Wave Lett., vol. 7, no. 6, pp. 168-170, Jun. 1997.

[19] L. Harle and L. Katehi, "A silicon micromachined four-pole linear phase filter," in IEEE Trans. Microw. Theory Tech., vol. 52, no. 6, pp. 15981607, Jun. 2004

[20] M. Stickel, P. Kremer, and G. V. Eleftheriades, "A millimeter-wave bandpass waveguide filter using a width-stacked silicon bulk micromachining approach," in IEEE Microw. Wireless Compon. Lett., vol. 16, no. 4, pp. 209-211, Apr. 2006.

[21] B. Pan, Y. Li, M. M. Tentzeris, and J. Papapolymerou, "Surface micromachining polymer-core-conductor approach for high-performance millimeter-wave air-cavity filters integration," in IEEE Trans. Microw. Theory Tech., vol. 56, no. 4, pp. 959-970, Apr. 2008.

[22] S. Song and K.-S. Seo, "A W-band air-cavity filter integrated on a thinfilm substrate," in IEEE Microw.Wireless Compon. Lett., vol. 19, no. 4 , pp. 200-202, Apr. 2009

[23] S. Song, C-S. Yoo, and K-S. Seo, "W-Band bandpass filter using micromachined air-cavity resonator with current probes," in IEEE Microwave and Wireless Components Letters, vol.20, no. 4, pp. 205207, Apr. 2010

[24] J.-S. Hong and M.J. Lancaster, Microstrip filters for RF/microwave applications, Wiley series in microwave and optical engineering, New York, 2001.

[25] D. G. Swanson, "Narrow-band microwave filter design," IEEE Microwave Magazine, vol.8, no.5, pp.105-114, Oct. 2007.

[26] G.L. Matthaei, L. Young and E.M.T. Jones, Microwave filters, impedance-matching networks and coupling structures, McGraw-Hill, New York, 1964.

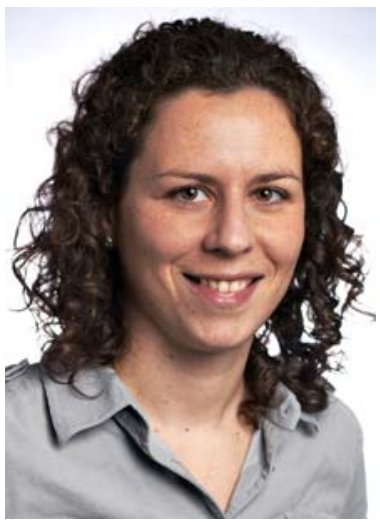

Esperanza Alfonso Alós received the M.S. and Ph.D. degrees in electrical engineering from the Universidad Politécnica de Valencia, Valencia, Spain, in 2004 and 2011, respectively.

From 2004 to 2010, she was a Research Assistant at the Institute of Telecommunications and Multimedia Applications (iTEAM), Universidad Politécnica de Valencia. During the autumns of 2007 and 2008, she was on leave at the Antenna Group, Chalmers University of Technology, Gothenburg, Sweden, where she was studying new quasi-TEM waveguides based on periodic or artificial surfaces. In 2011, she joined the Antenna Group at Chalmers University of Technology, where she is currently a Postdoc Researcher. Her research interests includes computational electromagnetics, antenna design and gap waveguides.

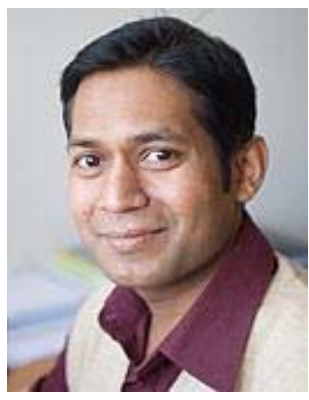

Ashraf Uz Zaman was born in Chittagong, Bangladesh. He received his BSc. in Electrica and Electrical Engineering from Chittagong University of Engineering and Technology, Bangladesh. In 2007, he received his MSc degree from Chalmers University of Technology, Sweden. At present, he is with the Communication, Information Theory and Antenna division of same university and is working towards his $\mathrm{PhD}$. His main research interest includes millimeter and sub millimeter waveguide technology, frequency selective surfaces, microwave passive components, slot array antennas, integration of MMIC with the antennas etc.

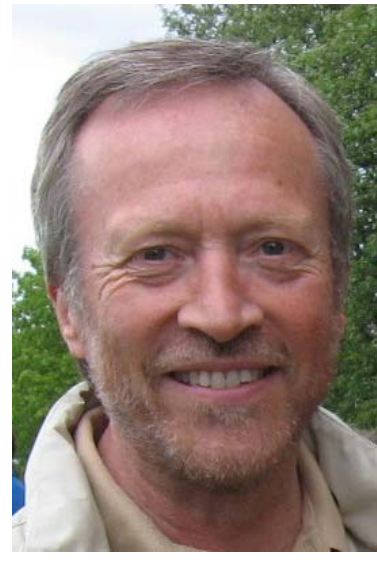

Per-Simon Kildal (M'82-SM'84-F'95) has been Professor at Chalmers University of Technology, Gothenburg, Sweden since 1989. He has authored an antenna texbook, and more than 110 journal articles and letters in IEEE or IET journals. He has designed two very large antennas, including the Gregorian dualreflector feed of Arecibo radiotelescope. He has invented several reflector antenna feeds, the latest being the so-called "Eleven antenna". He is the originator of the concept of soft and hard surfaces, recently resulting in the gap waveguide, a new low-loss metamaterial-based transmission line advantageous in particular above $30 \mathrm{GHz}$. His research group has pioneered the reverberation chamber into an accurate measurement tool for antennas and wireless terminals subject to Rayleigh fading.

Prof Kildal received two best paper awards for articles published in the IEEE Transaction on Antennas and Propagation, and he is the recipient of the 2011 Distinguished Achievements Award of the IEEE Antennas and Propagation Society 